\title{
Inflammatory Myofibroblastic Tumor 12 Years After Treatment for Synovial Sarcoma: A Case Report
}

\author{
Aadit Shah $\mathbb{D}^{\prime}$ \\ Eduard Pey ${ }^{2}$ \\ Justice U Achonu' \\ Ji Dong K Bai ${ }^{3}$ \\ Fazel Khan' \\ 'Department of Orthopaedics, Stony \\ Brook University Hospital, Stony Brook, \\ NY, USA; '2 Stony Brook University \\ Renaissance School of Medicine, Stony \\ Brook, NY, USA; ${ }^{3}$ Department of \\ Pathology, Stony Brook University \\ Hospital, Stony Brook, NY, USA
}

\begin{abstract}
Inflammatory myofibroblastic tumors (IMTs) are mesenchymal neoplasms most seen in the abdominopelvic region, lung, and retroperitoneum; and less commonly seen in virtually any other site. We report a case of two lower limb masses consistent with diagnosis of IMTs. This is a 39-year-old woman with a history of right lower extremity popliteal fossa synovial sarcoma diagnosed 12 years prior and treated with chemotherapy, surgery, and radiation. She presented with two new - one anterior and one posterior - right thigh masses. Biopsies of the lesions demonstrated low-grade inflammatory spindle cell lesions at both sites. Wide resection was performed for both masses and further characterization of the surgical specimens was most consistent with IMT. At follow-up, the patient is well with no signs of recurrence 19 and 7 months postoperative to the resection of the anterior and posterior thigh masses, respectively. This case represents the first reported IMTs occurring as late as 12 years after primary cancer treatment, and the first occurring after synovial sarcoma.
\end{abstract}

Keywords: inflammatory myofibroblastic tumor, radiation, resection, synovial sarcoma

\section{Introduction}

Inflammatory myofibroblast tumor (IMT) is a neoplasm of mesenchymal origin, most typically affecting children and young adults, with a predilection for visceral soft tissues. $^{1,2}$ The term IMT emerged as a discrete entity from the benign morphological group termed "inflammatory pseudotumors (IPT)" roughly two decades ago. Its evolving, and at times, inconsistently applied nosology makes it difficult or impossible to tabulate the total number of reported cases. However, data from more recent case reports and two notable case series with 38 and 84 cases, respectively, underlie the present academic understanding., ${ }^{3,4}$ IMT is most commonly seen in the lung, abdominopelvic region, and retroperitoneum, but may also be seen in virtually any site. ${ }^{2}$ Clinical presentation is typically due to the mass itself, or nonspecific symptoms resulting from the tumor's mass effect such as abdominal pain, gastrointestinal complaints, cough, or chest pain. $^{4-6}$ A constitutional syndrome has also been described in $15-30 \%$ of the patients, consisting of fever, weight loss, and malaise, with laboratory evaluation revealing microcytic anemia, increased erythrocyte sedimentation rate, thrombocytosis, and/or polyclonal hypergammaglobulinemia. ${ }^{3,4}$ Prognosis is influenced by a site-dependent 2 $25 \%$ recurrence rate. ${ }^{4,7,8}$ Additionally, rare distant metastasis is documented in 21 cases, most commonly to lung and brain, followed by liver and bone., ${ }^{2,3,9-20}$
Correspondence: Aadit Shah

Stony Brook University Hospital, Department of Orthopaedics, I0I Nicolls Road, HSC T- 18 - 089, Stony Brook, NY II794, USA

Tel +|-63|-786-9484

Email aadit.shah@stonybrookmedicine. edu 
Histologic characterization of IMT is classically characterized by spindle cell proliferation in a myxoid to collagenous stroma, with a prominent inflammatory infiltrate of mostly plasma cells and lymphocytes. ${ }^{2}$ Three basic histological patterns have been defined and are often seen in combination within the same tumor: a myxoid/vascular pattern, a compact spindle cell pattern, and a hypocellular fibrous pattern. ${ }^{4}$ Rearrangements of the $A L K$ (anaplastic lymphoma kinase) gene involving constitutive expression of its product have been documented, with ALK rearrangements and expression shown in approximately $50 \%$ of IMTs by both fluorescence in situ hybridization (FISH) and immunohistochemistry (IHC), respectively. ${ }^{21}$ ROS1 gene rearrangements have been identified most often in IMTs affecting children, many of these IMTs also demonstrate kinase fusions, which are often detected with immunohistochemistry or FISH studies. There have also been cases of IMTs that lack FISH abnormalities, and potential FISH and IHC assay issues may arise yielding false negatives. Kinase fusion negative IMTs lack these FISH abnormalities, making it more difficult to detect gene rearrangements such as ROS1, RET, NTRK, and PDGFRbeta. ${ }^{22,23}$ This case has been demonstrated previously in an 18-year-old woman who had an ALKnegative lung IMT. ${ }^{24}$ Another case study demonstrated detection of CDK4 and MDM2 double amplification in IMT tumor immunohistochemistry. ${ }^{25}$ Histological diagnosis of IMT carries a differential diagnosis including spindle cell sarcoma, spindle cell melanoma, sarcomatous carcinomas, dedifferentiated liposarcoma, gastrointestinal stromal tumors, dendritic cell neoplasms, desmoid fibromatosis, nodular fasciitis, and IPT. Of note, IMT is particularly a diagnosis of exclusion middle-aged or older adults, and when seen in skin or somatic soft tissue. ${ }^{2}$

Our case, accordingly, is one of these hard-fought diagnoses of exclusion: IMT near the knee in a 39-yearold woman, interestingly occurring 12 years after chemotherapy, surgical resection and radiation for a synovial sarcoma at the knee.

\section{Case Presentation}

Our patient is a 39 -year-old woman with a past medical history of right lower extremity popliteal fossa synovial sarcoma that was diagnosed in 2007 and treated with chemotherapy: 3 cycles pre- and 3 cycles post-surgery with Adriamycin, Ifosfamide, and Mesna (AIM); radiation; and surgery; as well as monoclonal gammopathy of undetermined significance (MGUS) followed with routine positron emission tomography/computed tomography
(PET/CT) scans, Sjogren's syndrome, and rheumatoid arthritis, who presented to the orthopaedic oncology clinic at our institution for evaluation of two newly discovered right thigh masses.

She was well-appearing and ambulated without any assistive devices. She had two palpable masses-one in the anterior thigh and the other in the posterior thigh. The skin overlying the anterior mass was intact, while the skin over the posterior one had a surgical scar with extensive fibrous and scar tissue likely from her previous radiation. She had a reduced range of motion of the knee, from 4 to 100 degrees, and no palpable lymphadenopathy. The remainder of the examination was normal.

Her diagnostic assessment revealed anterior and posterior subcutaneous soft tissue masses of the thigh. PET/CT studies revealed both tumors with standard uptake values (Figure 1). Several small, mildly hypermetabolic lymph nodes within the right external iliac and inguinal region were also identified, with the largest measuring $9 \mathrm{~mm}$ consistent with reactive lymph nodes.

Ultrasound-guided core biopsies were taken of the anterior and posterior thigh masses by interventional radiology. Both resulted in a similar histological picture spindle cell, myxoid lesion with a mixed inflammatory infiltrate. The cellular lesions consisted of low-grade myofibroblastic cells with associated myxoid matrix and a mixed inflammatory infiltrate, with neutrophils, plasma cells, and occasional eosinophils. There also was some free hemorrhage. The spindle cells stained positively for smooth muscle actin and negatively for ALK1, S100, CD99, cytokeratin 7, BCL-2, EMA, CD56, CD34. The plasma cells had no significant expression of IgG4. FISH analysis for SS18-SSX, a gene rearrangement for synovial sarcoma, was negative. Essentially, no definitive diagnosis was made.

Since the imaging-guided biopsies did not result in definitive diagnosis, we decided to perform an open biopsy with excision of the masses. Because of the presence of extensive dense and fibrous tissue posteriorly from previous surgery as well radiation, it was decided to operate in a staged fashion starting with the anterior mass. The anterior mass was excised and sent to pathology for evaluation. On initial evaluation, pathologists identified the features of the mass most closely resembling IMT and nodular fasciitis. FISH analysis later performed was negative for USP6 (17p13), a gene rearrangement found in $85-90 \%$ of the nodular fasciitis cases. The open biopsy and excision of the anterior mass was complicated by delayed wound healing, and 

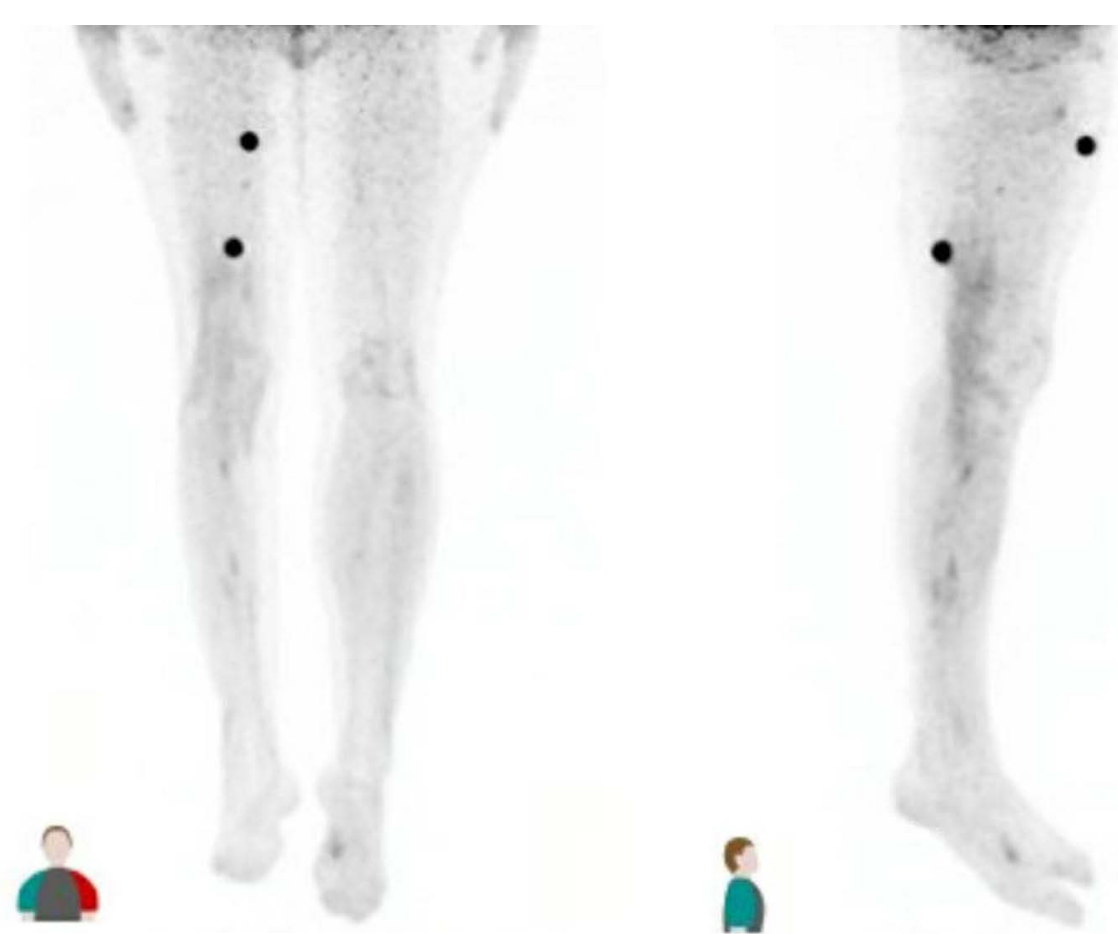

Figure I PET/CT image showing hyperdense lesions in the anterior and posterior right thigh. Views are anterior (left) and right lateral (right).

resection of the second, posterior mass was postponed until complete healing of this anterior wound.

During this period, a surveillance MRI performed showed an interval enlargement of the posterior thigh mass (Figure 2). There was increased infiltration of the subcutaneous tissue surrounding the lesion. Following this MRI and once the incision from the anterior mass had healed, it was decided to proceed with wide resection of the posterior thigh mass (Figure 3). The definitive wound closure procedure for this resection was performed by plastic surgery. Pathologic evaluation of the posterior mass like the anterior mass resulted in IMT and nodular fasciitis as being the top of the differential with FISH analysis showing negative USP6 rearrangement. In a multicenter discussion about the pathology, the highest diagnosis on the differential was IMT.

The patient has been seen up to 7 months post-op after resection of the posterior thigh mass (19 months after resection of the anterior mass). She has been doing well without any signs of recurrence of the disease.

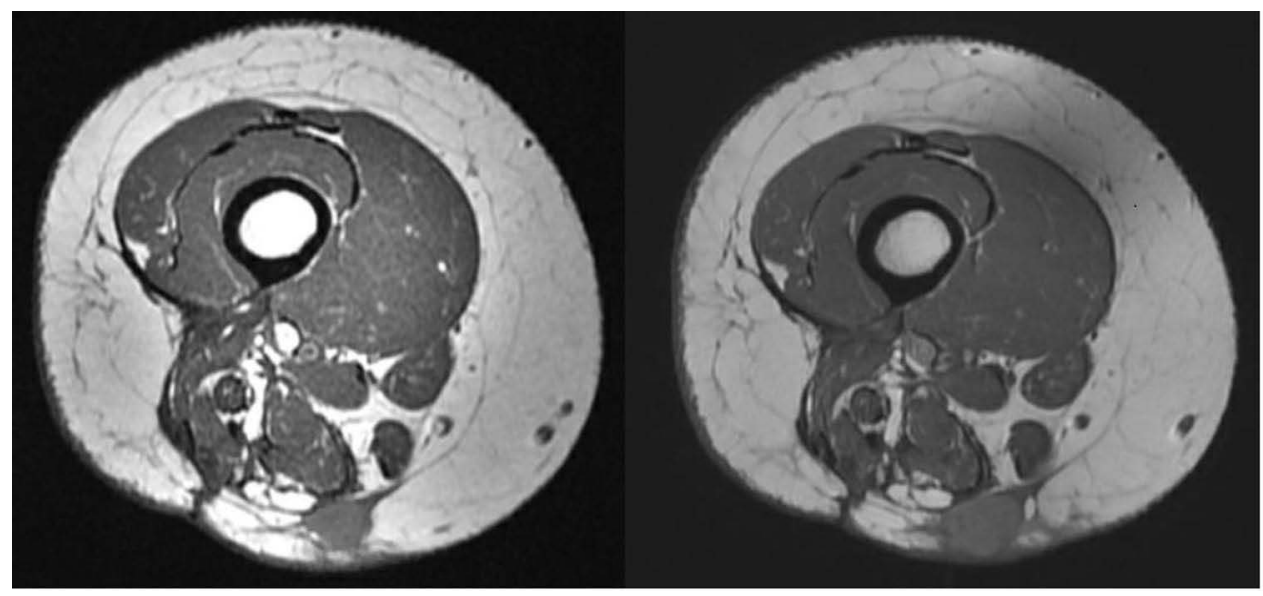

Figure $2 \mathrm{TI}$-weighted MRI showing interval enlargement of posterior thigh mass from 1.6 by $1.4 \mathrm{~cm}$ (left image) to 2.2 by $1.6 \mathrm{~cm}$ (right image), images were taken approximately 6 months apart. 


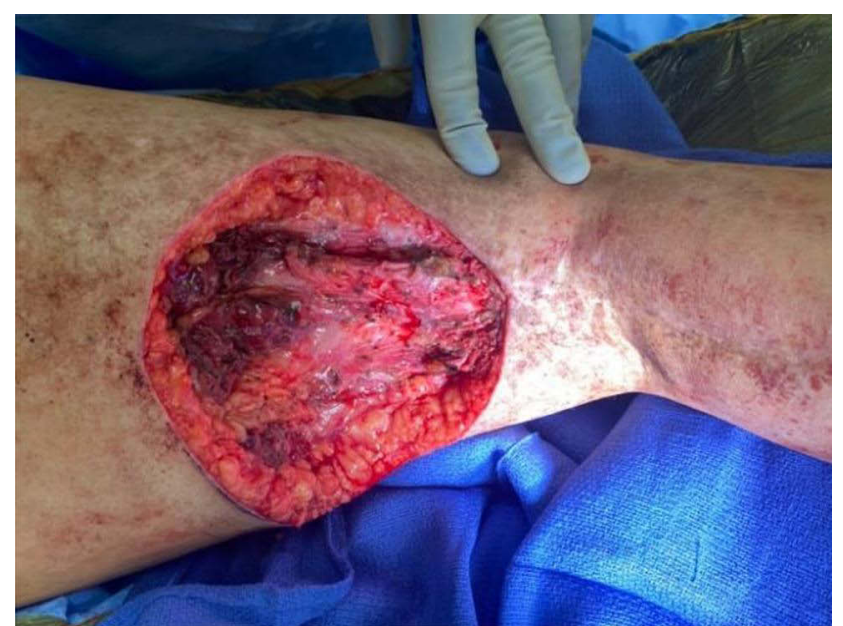

Figure 3 Surgical resection of the posterior thigh mass.

\section{Discussion}

After resection, chemotherapy, and radiation of a soft tissue sarcoma, $80 \%$ of the recurrences are detected within the first 2 years post-resection. ${ }^{26}$ Later recurrence may still occur, but this is rare. In this patient, 12 years had passed since diagnosis and treatment of her synovial sarcoma. Local recurrence rates following wide resection are $8-30 \%(60-90 \%$ for simple excision). ${ }^{26}$ Postoperative surveillance recommendations include periodic physical examination, imaging of the primary site, and chest imaging to rule out metastatic disease. ${ }^{26}$ Routine evaluation of this patient did not reveal any recurrence or metastasis. She was also followed with PET/CT scans for her history of MGUS, which did not reveal any disease. Therefore, when she was evaluated 12 years after her synovial sarcoma resection, there was a low probability that the lesions were recurrence/metastasis. Synovial sarcoma recurrence/ metastasis was thus excluded as a likely diagnosis, even considering its location right at the site of prior radiation. After initial biopsy of the two masses, negativity FISH for SS18SSX of biopsy specimens further supported the diagnostic exclusion of recurrent synovial sarcoma, as up to $97 \%$ of the synovial sarcomas demonstrate this gene fusion. ${ }^{27}$

Histopathologic interpretation of the anterior thigh mass was characterized by its storiform cellular myofibroblastic pattern (Figure 4), with mixed inflammatory infiltrate of plasma cells, macrophages, neutrophils and some multinucleated giant cells. Collections of lymphocytes were also noted in the periphery of the lesion. Consistent with nodular fasciitis' higher incidence, as well as IMT being a diagnosis of exclusion, initial interpretation of this lesion was as a nodular fasciitis. However, once FISH came back negative for USP6 this changed the scenario as this test has a positive predictive value of $100 \%$ and a negative predictive value of
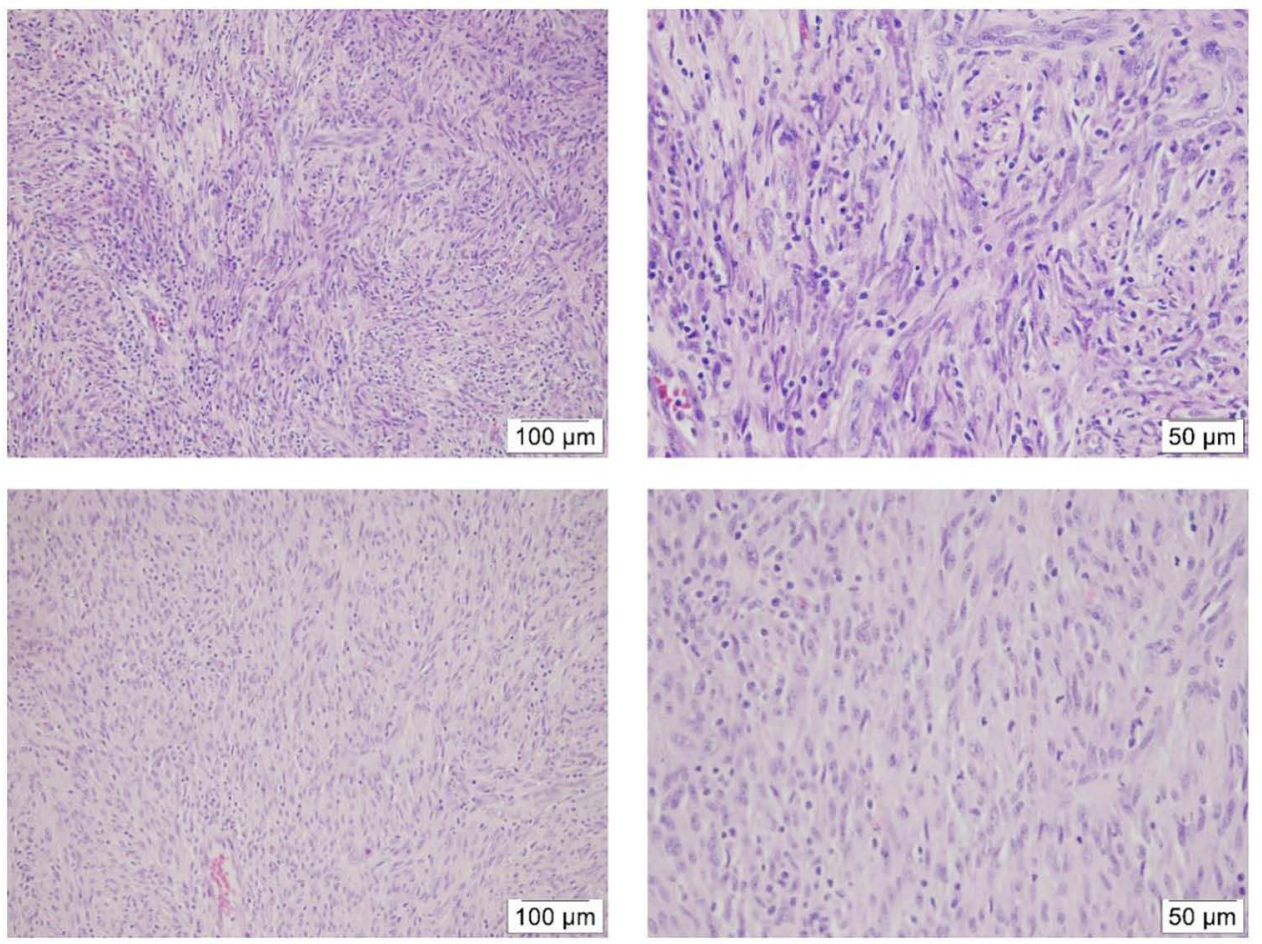

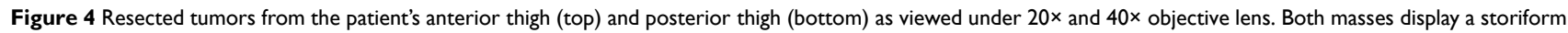
architecture of spindle cells with ovoid nuclei in collagenous stroma, most consistent with the known "compact spindle cell pattern" of IMT. 
$90 \%$, with $10-15 \%$ of the cases of nodular fasciitis being negative for the gene rearrangement. ${ }^{28}$ IgG4-related disease was also suspected due to her history of MGUS, but on immunohistochemistry, the plasma cells that were present did not significantly express IgG4.

The posterior mass was histologically similar to the former, and with similar FISH negatives for SS18/SSX and USP6, was evaluated as most consistent with inflammatory myofibroblastic tumor. The lesion tested negative for ALK1, a known marker for IMT. Only 50\% of IMTs are said to carry this marker, and moreover, most of those are in younger patients. $^{2}$ It is important to note, IMTs can be negative for a kinase fusion on immunohistochemistry/FISH and still be the diagnosis. Our patient being 39 years old places her at the upper end of the bell curve; therefore, the probability of identifying ALK1 in an IMT lesion is likely $<50 \%$.

It has been demonstrated that IMT can result from surgical treatment, post-chemotherapy, and post-radiation treatment. $^{29,30}$ A multicenter study has reported on IMTs that have developed locally, and multifocally as metastatic disease; IMT has developed as the second tumor in two prior cases as reported on by the European pediatric Soft Tissue Sarcoma Study Group (EpSSG) in a multicenter study. ${ }^{31}$ In both cases, however, the primary sarcomas were treated with chemotherapy and radiotherapy, and the IMT developed outside of the treatment area within 1 and 5 years. ${ }^{31}$ The tumor has also been shown to develop as much as 12-19 years later. ${ }^{5}$ This is a unique case as the IMT developed locally within the radiation therapy field 12 years post-surgery, postchemotherapy, and post-radiation. This tumor has been demonstrated to have both benign and aggressive features. It is described as a tumor with indeterminate biological potential: the recurrence rate is $25 \%$ and some can become malignant. ${ }^{32}$ In the case of this IMT, its features were benign and described as low-grade.

IMT is a rare neoplasm overall, with rudimentary epidemiology supporting 150-200 new cases per year in the United States. $^{31}$ Its post-surgical/traumatic and postradiation development is rare; especially locally and multifocally. With evidence of these tumors expressing benign and potentially aggressive behavior, 5-year event-free survival (EFS) and overall survival (OS) have been reported to be $82.9 \%$ and $98.1 \%$, respectively. ${ }^{31}$ IMT has also responded to systemic therapy including chemotherapy and radiation treatment in select cases. ${ }^{31,33}$ The rare ROS1rearranged IMTs have been found to respond to crizotinib, an antibody that has demonstrated significant reduction in tumor size. (Mai) Trabectedin, a marine-derived anticancer agent, is a microenvironment-targeting drug that is currently being investigated. It exerts a cytotoxic effect against the promoters of tumor-related inflammation. (Recine) A similar case report involving an IMT in the posterior thigh found no recurrence at 2 years, but reported the presence of a potential red herring: a postoperative cyst. ${ }^{34}$ Regardless, early diagnosis and prompt surgical treatment is warranted, and should be pursued when possible.

\section{Conclusion}

As introduced earlier, IMT is a difficult diagnosis with a history of mischaracterization and misrepresentation in the literature and is a diagnosis of exclusion-particularly in a patient such as ours where a mass is seen in skin or soft tissue. The lack of positive and supportive molecular stains makes this patient's lesions difficult to classify; however, its closest differential diagnosis given this histological presentation, nodular fasciitis, has been quite strongly ruled out with the absence of the USP6 gene rearrangement, and histological appearance is consistent with IMT. If both lesions are IMT, this would be the first published case to have occurred as far out as 12 years after primary cancer treatment with chemotherapy and radiation, and the first to have occurred secondary to synovial sarcoma. We endorse the necessity of early diagnosis and prompt surgical treatment of tumors presenting as in our patient.

\section{Consent for Publication}

The patient was aware of their participation in the case report and informed consent was obtained from the patient for publication. Institutional approval was not required to publish the case details.

\section{Acknowledgments}

No support was received for this manuscript. Investigation was performed at Stony Brook University Hospital, Stony Brook, New York, USA.

\section{Disclosure}

FK reports patent pending for computer navigation type use in bone sarcomas, outside of this work. The authors declare no other potential conflicts of interest.

\section{References}

1. Surabhi VR, Chua S, Patel RP, Takahashi N, Lalwani N, Prasad SR. Inflammatory myofibroblastic tumors: current update. Radiol Clin North Am. 2016;54(3):553-563. doi:10.1016/j.rcl.2015.12.005 
2. Gleason BC, Hornick JL. Inflammatory myofibroblastic tumours: where are we now? J Clin Pathol. 2008;61(4):428-437. doi:10.1136/jcp.2007.049387

3. Meis JM, Enzinger FM. Inflammatory fibrosarcoma of the mesentery and retroperitoneum. A tumor closely simulating inflammatory pseudotumor. Am J Surg Pathol. 1991;15(12):1146-1156. doi:10.1097/00000478-199112000-00005

4. Coffin CM, Watterson J, Priest JR, Dehner LP. Extrapulmonary inflammatory myofibroblastic tumor (inflammatory pseudotumor). A clinicopathologic and immunohistochemical study of 84 cases. Am J Surg Pathol. 1995;19(8):859-872. doi:10.1097/00000478199508000-00001

5. Pettinato G, Manivel JC, De Rosa N, Dehner LP. Inflammatory myofibroblastic tumor (plasma cell granuloma). Clinicopathologic study of 20 cases with immunohistochemical and ultrastructural observations. Am J Clin Pathol. 1990;94(5):538-546. doi:10.1093/ ajcp/94.5.538

6. Spencer H. The pulmonary plasma cell/histiocytoma complex. Histopathology. 1984;8(6):903-916. doi:10.1111/j.1365-2559.1984. tb02409.x

7. Cerfolio RJ, Allen MS, Nascimento AG, et al. Inflammatory pseudotumors of the lung. Ann Thorac Surg. 1999;67(4):933-936. doi:10.1016/S0003-4975(99)00155-1

8. Janik JS, Janik JP, Lovell MA, Hendrickson RJ, Bensard DD, Greffe BS. Recurrent inflammatory pseudotumors in children. $J$ Pediatr Surg. 2003;38(10):1491-1495. doi:10.1016/S00223468(03)00501-3

9. Myint MA, Medeiros LJ, Sulaiman RA, Aswad BI, Glantz L. Inflammatory pseudotumor of the ileum. A report of a multifocal, transmural lesion with regional lymph node involvement. Arch Pathol Lab Med. 1994;118(11):1138-1142.

10. Coffin CM, Hornick JL, Fletcher CDM. Inflammatory myofibroblastic tumor: comparison of clinicopathologic, histologic, and immunohistochemical features including ALK expression in atypical and aggressive cases. Am J Surg Pathol. 2007;31(4):509-520. doi:10.1097/01.pas.0000213393.57322.c7

11. Donner LR, Trompler RA, White RR. Progression of inflammatory myofibroblastic tumor (inflammatory pseudotumor) of soft tissue into sarcoma after several recurrences. Hum Pathol. 1996;27 (10):1095-1098. doi:10.1016/S0046-8177(96)90291-9

12. Biselli R, Ferlini C, Fattorossi A, Boldrini R, Bosman C. Inflammatory myofibroblastic tumor (inflammatory pseudotumor): DNA flow cytometric analysis of nine pediatric cases. Cancer. 1996;77(4):778-784. doi:10.1002/(SICI)1097-0142(19960215) 77:4<778::AID-CNCR25>3.0.CO;2-X

13. Malhotra V, Tatke M, Malik R, et al. An unusual case of plasma cell granuloma involving lung and brain. Indian J Cancer. 1991;28 (4):223-227.

14. Hasegawa SL, Schofield DE, Fletcher CDM. Inflammatory myofibroblastic tumor or inflammatory fibrosarcoma? AJSP Rev Rep. 1998;3 (3):128-134.

15. Hagenstad CT, Kilpatrick SE, Pettenati MJ, Savage PD. Inflammatory myofibroblastic tumor with bone marrow involvement. A case report and review of the literature. Arch Pathol Lab Med. 2003;127(7):865-867. doi:10.5858/2003-127-865-IMTWBM

16. Trojan A, Stallmach T, Kollias S, Pestalozzi BC. Inflammatory myofibroblastic tumor with CNS involvement. Onkologie. 2001;24 (4):368-372.

17. Watanabe K, Tajino T, Sekiguchi M, Suzuki T. Inflammatory myofibroblastic tumor (inflammatory fibrosarcoma) of the bone. Arch Pathol Lab Med. 2000;124(10):1514-1517. doi:10.5858/2000-1241514-IMTIFO

18. Petridis AK, Hempelmann RG, Hugo HH, Eichmann T, Mehdorn HM. Metastatic low-grade inflammatory myofibroblastic tumor (IMT) in the central nervous system of a 29 -year-old male patient. Clin Neuropathol. 2004;23(4):158-166.
19. Morotti RA, Legman MD, Kerkar N, Pawel BR, Sanger WG, Coffin CM. Pediatric inflammatory myofibroblastic tumor with late metastasis to the lung: case report and review of the literature. Pediatr Dev Pathol. 2005;8(2):224-229. doi:10.1007/s10024-0048088-5

20. Chun YS, Wang L, Nascimento AG, Moir CR, Rodeberg DA. Pediatric inflammatory myofibroblastic tumor: anaplastic lymphoma kinase (ALK) expression and prognosis. Pediatr Blood Cancer. 2005;45(6):796-801. doi:10.1002/pbc.20294

21. Coffin CM, Patel A, Perkins S, Elenitoba-Johnson KS, Perlman E, Griffin CA. ALK1 and p80 expression and chromosomal rearrangements involving 2p23 in inflammatory myofibroblastic tumor. Mod Pathol. 2001;14(6):569-576. doi:10.1038/modpathol.3880352

22. Antonescu CR, Suurmeijer AJ, Zhang L, et al. Molecular characterization of inflammatory myofibroblastic tumors with frequent ALK and ROS1 gene fusions and rare novel RET rearrangement. Am $J \quad$ Surg Pathol. 2015;39(7):957-967. doi:10.1097/PAS.00000 00000000404

23. Mai S, Xiong G, Diao D, Wang W, Zhou Y, Cai R. Case report: crizotinib is effective in a patient with ROS1-rearranged pulmonary inflammatory myofibroblastic tumor. Lung Cancer. 2019;128:101-104. doi:10.1016/j.lungcan.2018.12.016

24. Debonis SA, Bongiovanni A, Pieri F, et al. ALK-negative lung inflammatory myofibroblastic tumor in a young adult: a case report and literature review of molecular alterations. Medicine. 2021;100 (20):e25972. doi:10.1097/MD.0000000000025972

25. He J, Zhao X, Huang C, et al. Double amplifications of CDK4 and MDM2 in a gastric inflammatory myofibroblastic tumor mimicking cancer with local invasion of the spleen and diaphragm. Cancer Biol Ther. 2018;19(11):967-972. doi:10.1080/15384047.2018.1480290

26. Rothermundt C, Whelan JS, Dileo $\mathrm{P}$, et al. What is the role of routine follow-up for localised limb soft tissue sarcomas? A retrospective analysis of 174 patients. Br J Cancer. 2014;110(10):2420-2426. doi:10.1038/bjc.2014.200

27. Dos Santos NR, de Bruijn DR, van Kessel AG. Molecular mechanisms underlying human synovial sarcoma development. Genes Chromosomes Cancer. 2001;30(1):1-14. doi:10.1002/1098-2264(2000)9999:9999<::AID-GCC1056>3.0.CO;2-G

28. Shin C, Low I, Ng D, Oei P, Miles C, Symmans P. USP6 gene rearrangement in nodular fasciitis and histological mimics. Histopathology. 2016;69(5):784-791. doi:10.1111/his.13011

29. Vecchio GM, Amico P, Grasso G, Vasquez E, La Greca G, Magro G. Post-traumatic inflammatory pseudotumor of the breast with atypical morphological features: a potential diagnostic pitfall. Report of a case and a critical review of the literature. Pathol Res Pract. 2011;207 (5):322-326. doi:10.1016/j.prp.2011.01.009

30. Ilic V, Dunet V, Beck-Popovic M, Boubaker A. Pulmonary inflammatory myofibroblastic tumour after Hodgkin's lymphoma. BMJ Case Rep. 2014;2014:bcr2013202491. doi:10.1136/bcr-2013202491

31. Casanova M, Brennan B, Alaggio R, et al. Inflammatory myofibroblastic tumor: the experience of the European pediatric Soft Tissue Sarcoma Study Group (EpSSG). Eur J Cancer. 2020;127:123-129. doi:10.1016/j.ejca.2019.12.021

32. Hussong JW, Brown M, Perkins SL, Dehner LP, Coffin CM. Comparison of DNA ploidy, histologic, and immunohistochemical findings with clinical outcome in inflammatory myofibroblastic tumors. Mod Pathol. 1999;12(3):279-286.

33. Browne CP, Zeman-Pocrnich C, Dar AR, Wyllie B, Joseph M, Rare A. Case of inflammatory myofibroblastic tumor causing left pulmonary artery stenosis and successfully treated with localized radiotherapy in a patient with perinuclear antineutrophil cytoplasmic antibody vasculitis. Cureus. 2020;12(1):e6709.

34. Liu H, Lin J, Yang P, Shen H, Yang H. Whether inflammatory myofibroblastic tumor of the thigh relapses after surgical excision? Int J Clin Exp Med. 2015;8(7):11584-11588. 
Orthopedic Research and Reviews

Dovepress

\section{Publish your work in this journal}

Orthopedic Research and Reviews is an international, peer-reviewed, open access journal that focusing on the patho-physiology of the musculoskeletal system, trauma, surgery and other corrective interventions to restore mobility and function. Advances in new technologies, materials, techniques and pharmacological agents are particularly

welcome. The manuscript management system is completely online and includes a very quick and fair peer-review system, which is all easy to use. Visit http://www.dovepress.com/testimonials.php to read real quotes from published authors.

Submit your manuscript here: https://www.dovepress.com/orthopedic-research-and-reviews-journal 